\title{
On the stability of von Kármán rotating-disk boundary layers with radial anisotropic surface roughness
}

\author{
S.J. Garrett, ${ }^{1, a)}$ A.J. Cooper, ${ }^{2}$ J.H. Harris, ${ }^{2}$ M. Özkan, ${ }^{2}$ A. Segalini, ${ }^{3}$ and \\ P.J. Thomas ${ }^{2}$ \\ ${ }^{1)}$ Department of Engineering, University of Leicester, Leicester, LE1 7RH, \\ $U K$ \\ 2) School of Engineering, University of Warwick, Coventry, CV4 $7 A L$, \\ $U K$ \\ ${ }^{3)}$ Linné FLOW Centre, KTH Mechanics, SE-100 44, Stockholm, \\ Sweden
}

(Dated: 30 December 2015)

We summarise results of a theoretical study investigating the distinct convective instability properties of steady boundary-layer flow over rough rotating disks. A generic roughness pattern of concentric circles with sinusoidal surface undulations in the radial direction is considered. The goal is to compare predictions obtained by means of two alternative, and fundamentally different, modelling approaches for surface roughness for the first time. The motivating rationale being to identify commonalities and isolate results that might potentially represent artefacts associated with the particular methodologies underlying one of the two modelling approaches.

The most significant result of practical relevance obtained is that both approaches predict overall stabilising effects on the Type I instability mode of rotating disk flow. This mode leads to transition of the rotating-disk boundary layer and, more generally, the transition of boundary-layers with a cross-flow profile. Stabilisation of the Type 1 mode means that it may be possible to exploit surface roughness for laminar-flow control in boundary layers with a cross-flow component. However, we also find differences between the two sets of model predictions, some subtle and some substantial. These will represent criteria for establishing which of the two alternative approaches is more suitable to correctly describe experimental data when these become available.

\section{INTRODUCTION}

We recently reported first theoretical results investigating effects of distributed surface roughness on the convective stability of the rotating-disk boundary layer ${ }^{1}$. This analysis was based on the particular approach of Miklavčič \& Wang ${ }^{2}$ for modelling surface roughness and revealed stabilising roughness effects. In order to establish whether the predicted roughness effects arose as an artefact of the particular modelling approach of Ref. [2] we have attempted to reproduce them by means of an alternative, fundamentally different theoretical approach for the implementation of surface roughness. Here we summarize the results obtained from this alternative analysis and compare them to our original set of data from Ref. [1]. The goal is to highlight similarities and differences in the two data sets which we expect to become of significance in the context of interpreting future experimental data when they become available. We begin by briefly outlining the context of our overall programme.

The boundary-layer flow established over a rotating disk - in an infinite fluid environment that is at rest sufficiently far above the disk - is known as the von Kármán boundary layer ${ }^{3,4}$. It represents a typical, generic example of a general class of fully three-dimensional boundary layers that share the common, characteristic feature of what is known as a cross-flow velocity

\footnotetext{
a) stephen.garrett@le.ac.uk
} 
component ${ }^{4-6}$. Similar boundary layers are encountered in many applied contexts such as, for instance, on the blades of wind turbines or over the highly swept wings of aircraft.

All boundary layers with a cross-flow component display similar laminar-turbulent transition characteristics due to the existence of an inflection point on the cross-flow velocity profile $^{4-6}$. Hence, the results on roughness effects to be presented here are of general, direct practical relevance to all applied flow configurations where a boundary layer with a crossflow component is established. Our long-term goal being, as indicated above, to develop theoretical methods enabling the energetically-optimal design of surface-roughness that can be exploited, for boundary layers with a cross-flow component, in the context of new, passive drag-reduction techniques.

It has now been firmly established that - contrary to the classic belief - the interaction of boundary-layer flow with the right sort of roughness ${ }^{8}$ on surfaces can result in energetically beneficial, drag-reducing effects ${ }^{7-9}$. The challenge that remains is, however, to identify what represents the right sort of roughness that leads to such drag-reducing effects in any particular application and, moreover, to become able to reliably predict the roughness effects to be expected theoretically.

One fundamental, general strategy known to result in reduced drag is to control the laminar flow and delay its transition to turbulence. This method of the stabilisation of the boundary-layer flow exploits the fact that laminar flows are subject to smaller dissipative energy losses than turbulent flows. Our theoretical results discussed in Ref. [1] did predict such stabilising roughness effects on the rotating-disk boundary layer. However, these results were based on one particular theoretical approach of modelling roughness ${ }^{2}$ which has, as yet, not been tested experimentally. Therefore, it appeared necessary to explore and implement an alternative option for modelling surface roughness to investigate the robustness of our previous results and, thereby, generate confidence in their validity.

There exists an alternative, fundamentally different, method for modelling roughness than that introduced by Miklavčič \& Wang ${ }^{2}$. This alternative approach was suggested by Yoon, Hyun \& Park ${ }^{10}$. Henceforth we will refer to the two approaches as the MW and YHP models, respectively. Both models can be implemented to show how successively increasing roughness levels lead to deviations from the classic similarity solution for the flow over a smooth disk due to von Kármán ${ }^{3}$. It is these modified steady-flow base profiles which are underlying the subsequent linear stability analysis. In this context minor differences of the steady base flow have the potential to result in major discrepancies of the predicted overall stability charactersitics of the boundary layer.

The MW approach adopted in Ref. [1] models roughness empirically by replacing the usual no-slip boundary conditions with partial-slip conditions at the disk surface. This is achieved by introducing ad hoc slip factors in Newton's law of viscosity for the azimuthal and radial velocity component. Selecting different slip factors for each component enables modelling independent levels of roughness in the radial and azimuthal directions. We refer to the case where both slip factors are equal as isotropic roughness; whereas different values for the slip factors represent anisotropic roughness. The major weakness of the MW approach is that the slip factors have no apriori relation to any specific, geometric roughness height. Such a relation can only be established, if at all, through calibration procedures in connection with future experiments.

However, the YHP approach studied here models roughness by directly imposing a particular surface profile as a function of the radial position. Its drawback is, nevertheless, that it assumes rotational symmetry. The YHP approach therefore models roughness in the radial direction only and it can, hence, describe anisotropic roughness only. For our comparison with the MW model we have, therefore, selected the generic pattern of concentric grooves of a particular cross-sectional shape profile. However, the major advantage of the YHP approach over the MW model is that a specific geometric roughness height can be defined explicitly in terms of the amplitude and the wavelength of the surface undulations.

Due to the fact that the MW approach uses the slip factors, not related to any specific geometric roughness height, while the YHP model explicitly prescribes the roughness height it is not possible to make quantitative comparisons between the results obtained 
from both approaches. Currently only the comparison of qualitative results and trends can provide insight. However, this insight will ultimately enable formulating the criteria for deciding which modelling approach is more appropriate when experimental data will become available.

This paper proceeds as follows: In $\S I I$ we summarise the calculations of the steady boundary-layer flows over rotating disks with radial anisotropic surface roughness using the two models. We then investigate the convective stability properties of the two sets of resulting steady flows in $\S I I I$ and present neutral curves and critical Reynolds numbers. In $\S I V$ we present an analysis of the energy balance within the boundary layers arising from both models in order to extract possible underlying physical mechanisms behind the effects of roughness on the stability of the flows. Conclusions are then drawn in $\S \mathrm{V}$.

\section{THE STEADY FLOWS}

As discussed in $\S I$, two distinct approaches exist in the literature for modelling the steady boundary-layer flow over rotating disks with radial anisotropic surface roughness. The YHP model will be used with some modification to its original description ${ }^{10}$ and this warrants a detailed description in $\S I I$ A. The MW model will however be used without modification to its original presentation and we discuss it only briefly in $\S I I B$; full details are available elsewhere ${ }^{1,2}$.

\section{A. The surface-geometry model due to $\mathrm{YHP}^{10}$}

The surface of the disk is described by $s^{*}\left(r^{*}\right)=\delta^{*} \cos \left(2 \pi r^{*} / \gamma^{*}\right)$, with * indicating a dimensional quantity. The quantity $\delta^{*}$ is the amplitude of the surface variation from its mean value, $\gamma^{*}$ is the wavelength of the surface variation, and $r^{*}$ is the distance along the disk in the radial direction. The surface function can of course be altered to facilitate any required profile by changing the values of $\delta^{*}$ and $\gamma^{*}$, or indeed the functional form; however, the cosine function will be used throughout this study. The disk is considered to be rotating about its axis of symmetry at a constant rotation rate $\Omega^{*}$ and we formulate the analysis in the rotating frame. It is natural to consider this geometry in a cylindrical polar coordinate system $\left(r^{*}, \theta, z^{*}\right)$ in which the governing Navier-Stokes equations are well known. The steady-flow components in these directions are denoted $\left(u^{*}, v^{*}, w^{*}\right)$ and we assume a rotational symmetry such that the $\theta$-dependence can be neglected.

Note that the original formulation of this model ${ }^{10}$ is in the stationary frame of reference. This is in contrast to our choice of frame and we will necessarily find additional centrifugal terms in the analysis that follows. Furthermore, the original presentation considers the more general case of a rotating fluid in the far field and our current analysis corresponds to the particular case that their system parameter Ro is set to unity.

All dimensional quantities are scaled on a characteristic length-scale given by the boundary-layer thickness, $d^{*}=\sqrt{\nu^{*} / \Omega^{*}}$, where $\nu^{*}$ is the kinematic viscosity, and a velocity scale given by $r^{*} \Omega^{*}$. This leads to the Reynolds number $R e=r^{*} \Omega^{*} d^{*} / \nu^{*}=r$ and the non-dimensional coordinate system $(r, \theta, z)$. The surface function non-dimensionalises to

$$
s(r)=\delta \cos \left(\frac{2 \pi r}{\gamma}\right)
$$

This particular form of $s(r)$ gives two non-dimensional control parameters: $\delta$, the height of the roughness, and $\gamma$, the pitch of the roughness, both are expressed in units of boundarylayer thickness as a consequence of the spatial scalings. It is useful to define the aspect ratio $a=\delta / \gamma$ which we henceforth refer to as the roughness parameter within the YHP

model. The formulation used here is entirely consistent with the standard formulation of the rotating-disk problem in the literature and reduces to that previously used by Malik ${ }^{11}$ and Lingwood ${ }^{12}$, for example, when $a=0$. 
It is necessary to transform out the surface distribution before attempting to solve the governing equations. To this end we use a new coordinate system $(r, \theta, \eta)$ defined by the transformation $\eta=z-s(r)$. In this modified coordinate system the radial velocity, azimuthal velocity, axial velocity and pressure are transformed to, respectively,

$$
\begin{aligned}
U(r, \eta) & =u(r, z), \\
V(r, \eta) & =v(r, z), \\
\hat{W}(r, \eta) & =-s^{\prime}(r) u(r, z)+w(r, z), \\
P(r, \eta) & =p(r, z),
\end{aligned}
$$

where the prime denotes differentiation with respect to $r$. At this stage we make the boundary-layer assumption, $R e^{-1}<<1$, and set $W=\operatorname{Re} \hat{W}$ and $\zeta=R e \eta$. The boundarylayer equations are then obtained as

$$
\begin{aligned}
\frac{U}{r}+\frac{\partial U}{\partial r}+\frac{\partial W}{\partial \zeta} & =0 \\
U \frac{\partial U}{\partial r}+W \frac{\partial U}{\partial \zeta} & =-\frac{\partial P}{\partial r}+\operatorname{Res}^{\prime} \frac{\partial P}{\partial \zeta}+\left(1+s^{\prime 2}\right) \frac{\partial^{2} U}{\partial \zeta^{2}}+\frac{(r+V)^{2}}{r} \\
U \frac{\partial V}{\partial r}+W \frac{\partial V}{\partial \zeta} & =\left(1+s^{\prime 2}\right) \frac{\partial^{2} V}{\partial \zeta^{2}}-\frac{U(2 r+V)}{r}, \\
s^{\prime \prime} U^{2} & =s^{\prime} \frac{\partial P}{\partial r}-\operatorname{Re}\left(1+s^{\prime 2}\right) \frac{\partial P}{\partial \zeta}-s^{\prime} \frac{(r+V)^{2}}{r}
\end{aligned}
$$

Consistent with von Kármán's original analysis ${ }^{3}$, the pressure gradient in the radial direction is taken to be zero. The governing equations for the steady flow are obtained after introducing variables closely related to the von Kármán similarity variables,

$$
\begin{aligned}
f(r, \zeta) & =\frac{1}{r} U(r, \zeta), \\
g(r, \zeta) & =\frac{1}{r} V(r, \zeta), \\
h(r, \zeta) & =W(r, \zeta)
\end{aligned}
$$

and are stated as

$$
\begin{aligned}
2 f+r \frac{\partial f}{\partial r}+\frac{\partial h}{\partial \zeta} & =0, \\
r f \frac{\partial f}{\partial r}+h \frac{\partial f}{\partial \zeta}+\left(1+r \frac{s^{\prime} s^{\prime \prime}}{1+s^{\prime 2}}\right) f^{2} & =\left(1+s^{\prime 2}\right) \frac{\partial^{2} f}{\partial \zeta^{2}}+\frac{(1+g)^{2}}{1+s^{2}} \\
r f \frac{\partial g}{\partial r}+h \frac{\partial g}{\partial \zeta} & =\left(1+s^{\prime 2}\right) \frac{\partial^{2} g}{\partial \zeta^{2}}-2 f(1+g),
\end{aligned}
$$

These are subject to the boundary conditions

$$
\begin{aligned}
f(r, \zeta)=h(r, \zeta)=g(r, \zeta)=0 & \text { at } \zeta=0 \\
f(r, \zeta)=0, \quad g(r, \zeta)=-1 & \text { as } \zeta \rightarrow \infty
\end{aligned}
$$

which represent the no-slip and quiescent fluid conditions at all radial positions in this rotating frame of reference. Note that this PDE system reduces to the von Kármán system of ODEs in $\zeta$ when $s(r) \rightarrow 0$, as would be expected.

The presentation to this point has been consistent with the original description ${ }^{10}$ (albeit in the rotating frame) and we now proceed to discuss modifications to the model that are required in order to perform the stability analyses. Equations (3)-(6) can be solved, for example, using the commercially available NAG routine D03PEF. The routine is a PDE 
solver that reduces the PDEs to a system of ODEs in $\zeta$ using the method of lines, and solves the resulting ODEs using the backwards difference method. The solver uses an initial solution at $r=0$ to find the velocity profiles at the next increment of $r$ and marches forward. The velocity profiles at each $r$ are found using a grid between $\zeta=0$ and 20 .

The initial solution at $r=0$ is found from assuming that

$$
f(r, \zeta) \sim r F(\zeta), \quad g \sim r G(\zeta) \text { and } h \sim H(\zeta),
$$

as $r \rightarrow 0$, which results in the familiar set of von Kármán ODEs in $\zeta$. This approach is consistent with the series solution method described by Banks ${ }^{13}$ and applied more recently by Garrett \& Peake ${ }^{14}$ in a similar context for smooth surfaces.

The transformed flow field arising from the complete NAG solution across $(r, \zeta)$ is found to vary at two distinct spatial scales in the radial direction. At the scale characterised by $\gamma$, we have a response dependent on where $r$ is within the oscillatory cycle of the surface cosine function; that is, the flow fields at $r$ and $r+m \gamma$ (where $m$ is an integer) are identical. In addition to this oscillatory behaviour, we see a similarity-type solution scaling with $r$ (as per von Kármán ${ }^{3}$ ) at the larger spatial scale. For $\gamma<\mathcal{O}\left(10^{-1}\right)$, as is envisaged here, we argue that the small-scale response of the viscous flow will not occur in practice and it is a reasonable approximation to take a spatial average of the flow field over any complete cycle in $r$. This approach leaves only the similarity-solution variation within the averaged flow field, $(\bar{f}(\zeta), \bar{g}(\zeta), \bar{h}(\zeta))$. Note that overbars have been introduced to denote averaged quantities.

When the surface function $s(r)$ is oscillatory, as it is here, the spatial average acts to 'average away' the surface distribution, that is $\overline{s(r)}=0$ and so $\zeta \rightarrow z$. Under our approach the surface roughness is therefore seen to lead to a modified von Kármán flow, denoted $(\bar{f}(z), \bar{g}(z), \bar{h}(z))$.

Note that throughout this study we compute all spatially averaged quantities at 100 regularly spaced locations over one wavelength and the results have been confirmed to be independent of the starting radial position. Our results also show that the aspect ratio $a=\delta / \gamma$ determines the flow response and so, despite having two control parameters, we can work in terms of the single roughness parameter, $a$. A discussion of these aspects is given by Harris ${ }^{15}$.

Figure 1 shows the results from spatial averages of flows over one wavelength for $a=0$, 0.1, 0.2 and 0.3. The radial flow is shown in Figure 1(a) and roughness is seen to decrease the maximum radial velocity, $\max (\bar{f})$, within the boundary layer, i.e. roughness acts to reduce the wall jet. This is physically sensible as roughness would increase the friction holding back the base of the wall jet as it moves along the radius of the disk. For the azimuthal flow shown in Figure 1(b), roughness is seen to thicken the boundary layer through a widening of the profile; again, this is a physically sensible response. We note that the effect on the radial flow component arising from the YHP model is consistent with that obtained from the MW model ${ }^{1}$. However, for the wall-normal flow shown in Figure 1(c), roughness is here seen to increase the axial flow entrained, $\left|\bar{g}_{\infty}\right|$, into the boundary layer which is in direct contrast to the results from the alternative MW model. We return to the conflicting properties of the two models in the following section.

\section{B. The partial-slip model due to $\mathrm{MW}^{2}$}

Rather than imposing a particular mathematical form for the surface roughness, the MW approach assumes that roughness can be modelled by a modification of the no-slip conditions at the disk surface. In particular, the model assumes partial slip at the disk surface but is otherwise identical to the von Kármán formulation ${ }^{3}$; full details can be found elsewhere $^{1,2}$. The full MW model has two parameters $\eta$ and $\lambda$ (giving empirical measures of the roughness in the radial and azimuthal directions, respectively) that appear in the 


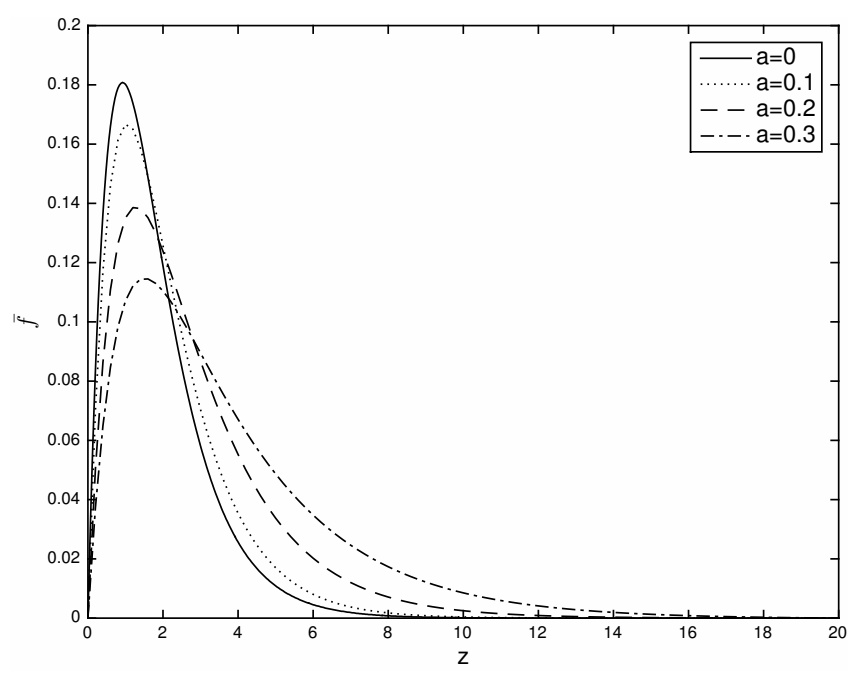

(a) $\bar{f}$-profile

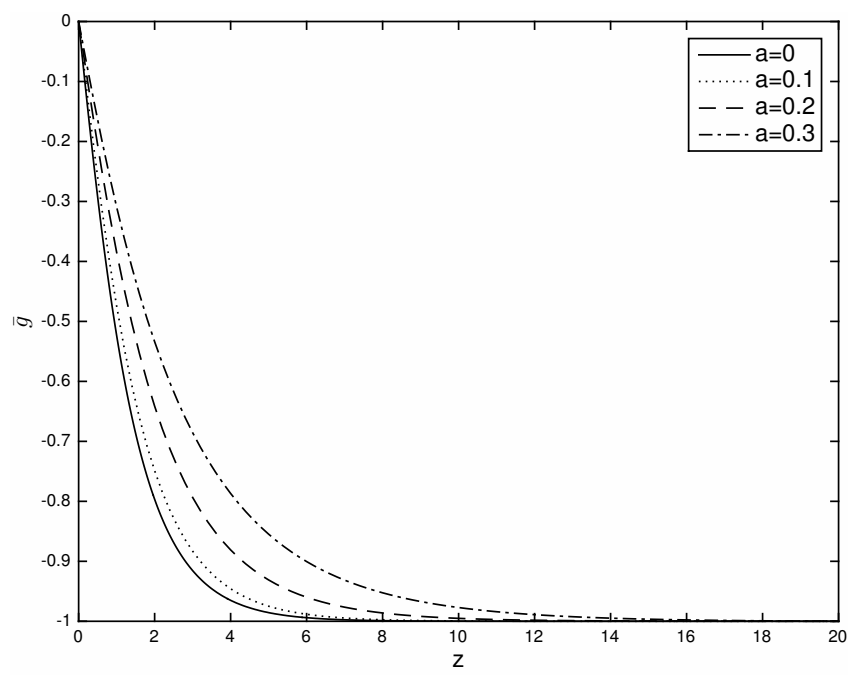

(b) $\bar{g}$-profile

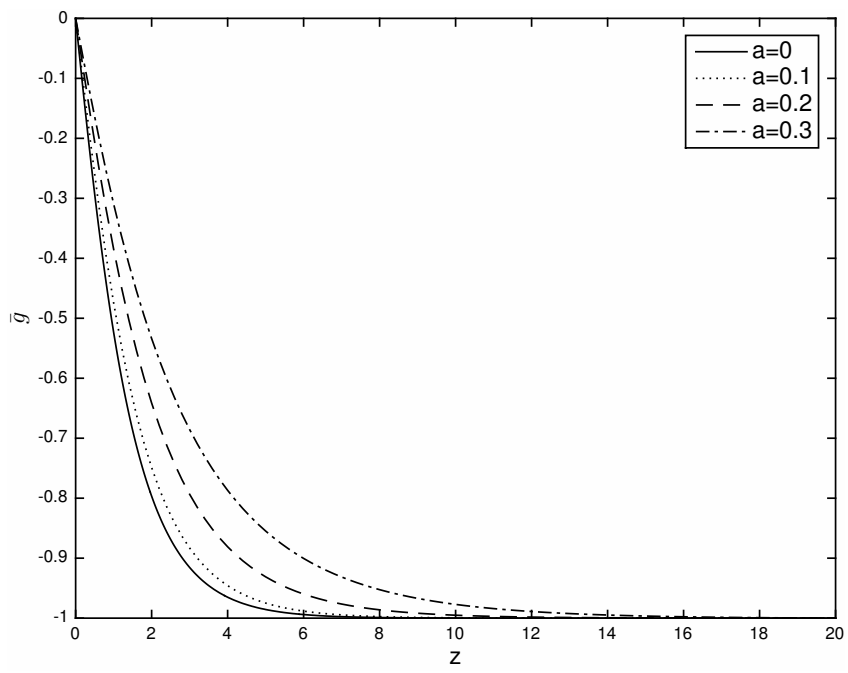

(c) $\bar{h}$-profile

FIG. 1: Steady-flow profiles resulting from spatial averages of the YHP model at various $a$. 
surface boundary conditions of the von Kármán ODEs,

$$
\begin{gathered}
\bar{f}(0)=\lambda \bar{f}^{\prime}(0), \\
\bar{g}(0)=\eta \bar{g}^{\prime}(0) .
\end{gathered}
$$

Here a prime denotes differentiation with respect to the normal spatial variable. Note that an overbar notation consistent with the presentation of the YHP model is used for the flow components throughout, although no spatial averaging is required in the MW approach. The empirical roughness parameters represent factors in Newton's law of viscosity and cannot, therefore, be associated with particular levels of roughness in practical applications without carefully produced calibration curves.

In this paper we are concerned with the particular case of anisotropic roughnesses in the radial direction, consistent with the capabilities of the YHP model, and so set $\lambda=0$. As with the YHP model, increased roughness in the MW model reduces the radial jet. In contrast to the YHP model, however, we see that the azimuthal profiles have a value at the disk surface that is progressively shifted backwards with increased roughness - this is a direct consequence of the boundary condition that underpins the approach - and the boundary layer is only marginally thickened. Furthermore, as mentioned previously, the wall-normal profiles show reducing axial entrainment with increased roughness which is in direct contrast to the response under the YHP model for the moderate levels of roughness investigated here. Despite both models predicting a reduced radial jet, the areas enclosed by the radial profiles are found to increase with roughness under the YHP model and decrease in the MW model. This area is a measure of the volume of fluid transported outwards in the radial direction and accounts for the different behaviour of the axial entrainment between the two models.

Given the different physical predictions arising from the two models and the empirical definition of roughness in the MW model, a direct quantitative comparison between 'equivalent' levels of roughness is not possible. Instead we proceed with a qualitative comparison of the effects of increasing roughness under both models. The particular values of $\eta$ used here are therefore reasonably arbitrary and we have opted to use the maximum value of the radial jet as a matching parameter. That is, for each value of $a$ in the YHP model, the value of $\eta$ in the MW model is chosen such that the maximum values of the radial wall jet, $\max (\bar{f})$, agree. Note that the azimuthal and wall-normal components can never be matched between the two models and we emphasise again that direct quantitative comparisons should not be made.

The paired parameter values are taken to be $a=0.1 \sim \eta=0.14, a=0.2 \sim \eta=0.57$ and $a=0.3 \sim \eta=1.18$ and the resulting flow profiles can be seen in Figure 2. Note that the azimuthal profiles are presented as $\bar{g}+1$ to separate the presentation of that component from the wall-normal component. Both models lead to the same von Kármán profiles for $a=\eta=0$, which can also be seen in Figure 1. Despite having identical values of the maximum wall jet, Figure 2 illustrates the significant differences in the flow profiles; these are further demonstrated in Table I. Note, in particular, that the YHP predictions for the azimuthal flow component approach the von Kármán solution for the smooth disks at low values of $z$ whereas the MW predictions consistently agree better with the von Kármán solution at higher values of $z$. The situation is more complex for the other two flow components. For instance, in the case of the wall-normal component, represented by $\bar{h}$, both the YHP and the MW models yield velocities higher than the von Kármán solution at lower values of $z$; but MW lies above von Kármán at higher $z$ whereas YHP lies below it. Despite both models leading to the same limiting behaviour of the radial component at the edge of the boundary layer, the YHP prediction has a much broader jet than both the MW and von Kármán solutions. Note moreover that the results of the MW model found here are entirely consistent with those found in our previous study ${ }^{1}$ at $\eta=0.25,0.5,0.75$ and 1.0. 


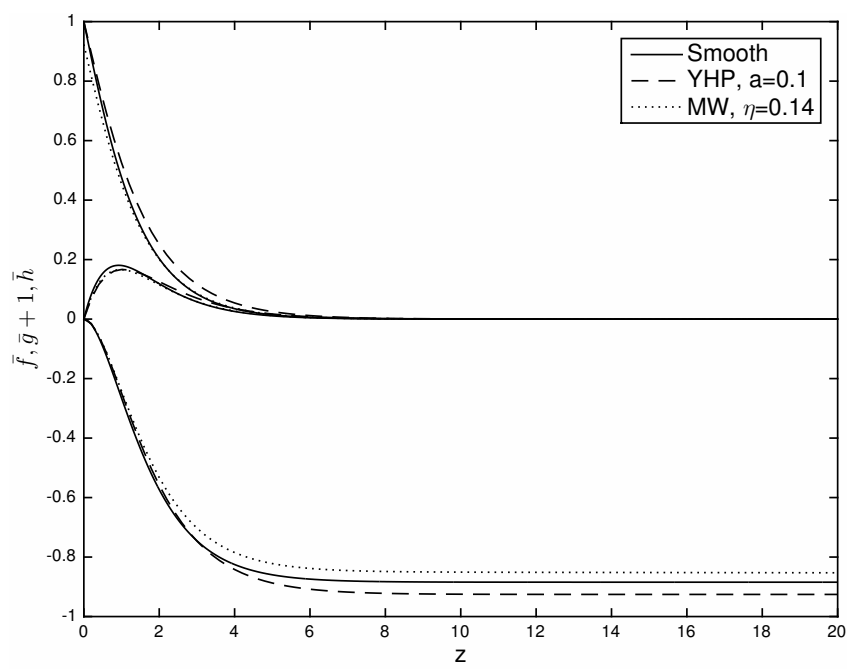

(a) $a=0.1, \eta=0.14$ such that $\max (\bar{f})=0.167$

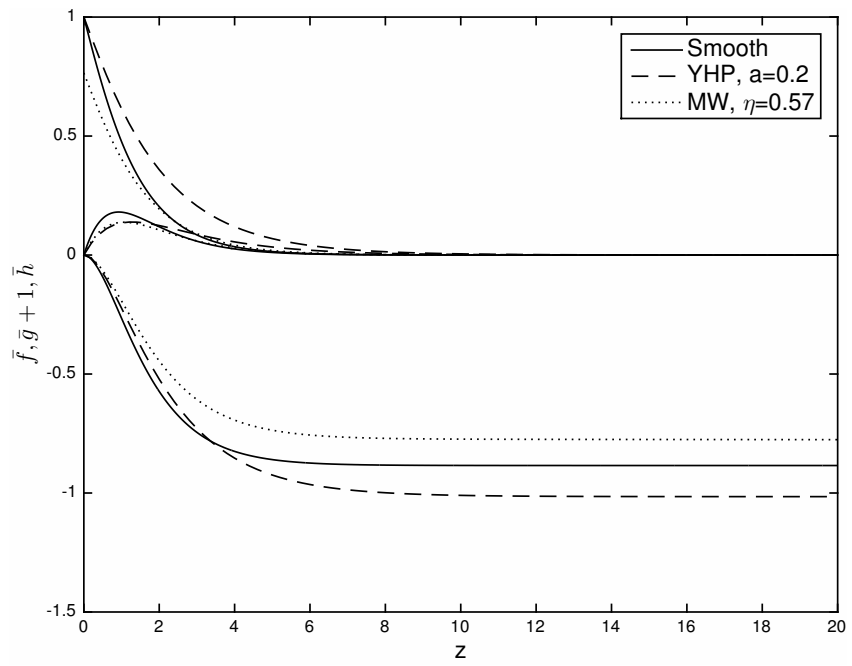

(b) $a=0.2, \eta=0.57$ such that $\max (\bar{f})=0.139$

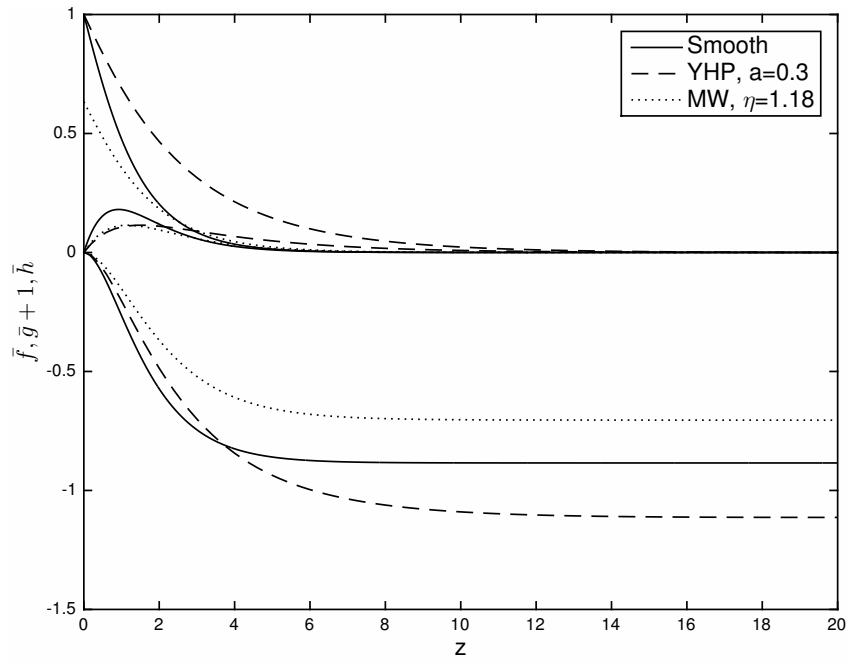

(c) $a=0.3, \eta=1.18$ such that $\max (\bar{f})=0.115$

FIG. 2: A comparison of the flow profiles resulting from the YHP and MW models with increasing roughness. The flows are paired by matching $\max (\bar{f})$. 


\begin{tabular}{lcccc} 
& \multicolumn{4}{c}{ YHP model } \\
& $\max (\bar{f})$ & $\bar{g}_{\infty}$ & $\int \bar{f} \mathrm{~d} z$ & $\int \bar{g} \mathrm{~d} z$ \\
\hline$a=0$ & 0.181 & -0.885 & 0.442 & 1.272 \\
$a=0.1$ & 0.167 & -0.926 & 0.463 & 1.451 \\
$a=0.2$ & 0.139 & -1.015 & 0.507 & 1.931 \\
$a=0.3$ & 0.115 & -1.113 & 0.555 & 2.622 \\
\multicolumn{5}{c}{ MW model } \\
\multicolumn{7}{c}{$\max (\bar{f})$} & $\bar{g}_{\infty}$ & $\int \bar{f} \mathrm{~d} z$ & $\int \bar{g} \mathrm{~d} z$ \\
\hline$\eta=0$ & 0.181 & -0.885 & 0.442 & 1.272 \\
$\eta=0.14$ & 0.167 & -0.850 & 0.425 & 1.222 \\
$\eta=0.57$ & 0.139 & -0.774 & 0.387 & 1.113 \\
$\eta=1.18$ & 0.115 & -0.704 & 0.352 & 1.012
\end{tabular}

TABLE I: A comparison of various properties of the steady flows resulting from the YHP and MW models with increasing roughness.

\section{CONVECTIVE INSTABILITY}

The two approaches used to calculate the steady flows in $\S I I$ both result in similarity solutions in the scaled physical space $(r, \theta, z)$. The resulting flows are therefore related to the von Kármán flow, and, importantly, the stability analyses of the rotating-disk flow presented elsewhere ${ }^{12,16}$ are directly applicable in this current study. Full details of the governing perturbation equations can be found in those references. Here it is sufficient to understand that we conduct a normal-mode analysis with perturbations of the form

$$
(\hat{u}, \hat{v}, \hat{w}, \hat{p})=(u(z), v(z), w(z), p(z)) e^{\mathrm{i}(\alpha r+\beta \operatorname{Re} \theta-\omega t)} .
$$

The wavenumber in the radial direction, $\alpha=\alpha_{r}+\mathrm{i} \alpha_{i}$, is complex, as required by the spatial convective analysis to be conducted; the frequency, $\omega$, and circumferential wavenumber, $\beta$, are real. It is assumed that $\beta$ is $O(1)$ and the integer number of complete cycles of the disturbance around the azimuth is $n=\beta R e$. We identify $n$ with the number of spiral vortices around the disk surface. Furthermore, the orientation angle of the vortices with respect to a circle centred on the axis of rotation is $\epsilon=\arctan (\beta / \alpha)$. The quantities $n$ and $\epsilon$ can be compared directly to experimental observations. Surface roughness is known to naturally excite and reinforce continuously disturbances that are fixed relative to the disk ${ }^{4}$. We therefore set $\omega=0$ and consider only disturbances that are stationary in our rotating frame.

The governing perturbation equations are solved using a Chebyshev polynomial discretisation method in the wall-normal direction to obtain solutions of the dispersion relation $D(\alpha, \beta ; \operatorname{Re},[a, \eta])=0$ with the aim of studying the occurrence of convective instabilities for various values of the roughness parameters. The use of the polynomials ensures a higher accuracy compared to standard finite differences methods with a similar discretisation. An exponential map is adopted to map the Gauss-Lobatto grid points used for the Chebyshev polynomials into the physical space: 100 points are therefore distributed between the disk surface $z=0$ and the top of the domain $z_{\max }=20$. The stability equations are written and solved in primitive variables at all the collocation points except the ones at the boundaries $\left(z=0\right.$ and $\left.z=z_{\max }\right)$, where the following boundary conditions are enforced

$$
\begin{aligned}
& u(z)=v(z)=w(z)=w^{\prime}(z)=0 \text { at } z=0 \\
& u(z)=v(z)=w(z)=p(z)=0 \text { at } z=z_{\max } .
\end{aligned}
$$

These conditions are identical to those used in our previous analysis of the MW model ${ }^{1}$. The straightforward implementation of the boundary conditions is a significant advantage of using primitive variables. 


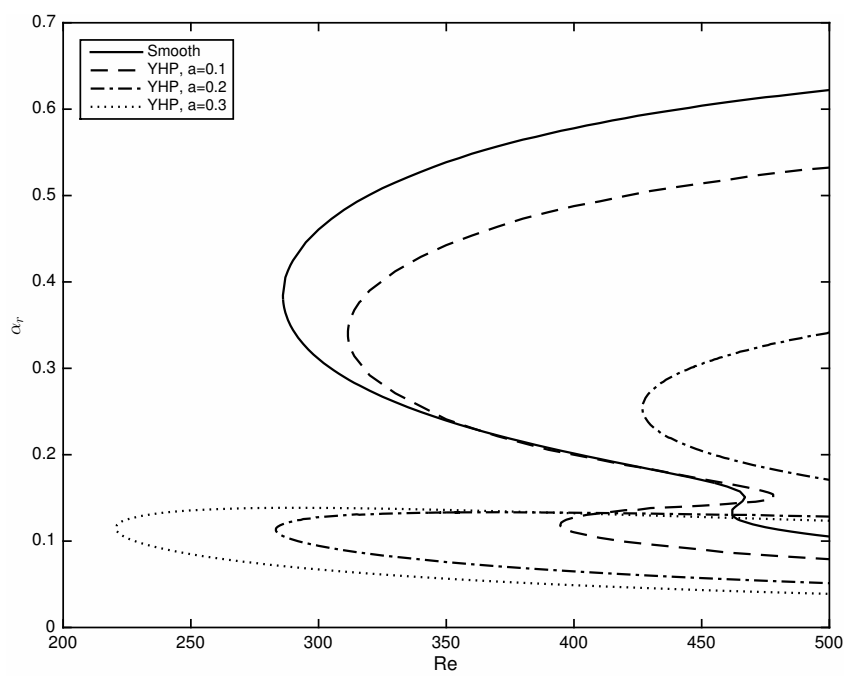

(a) YHP model

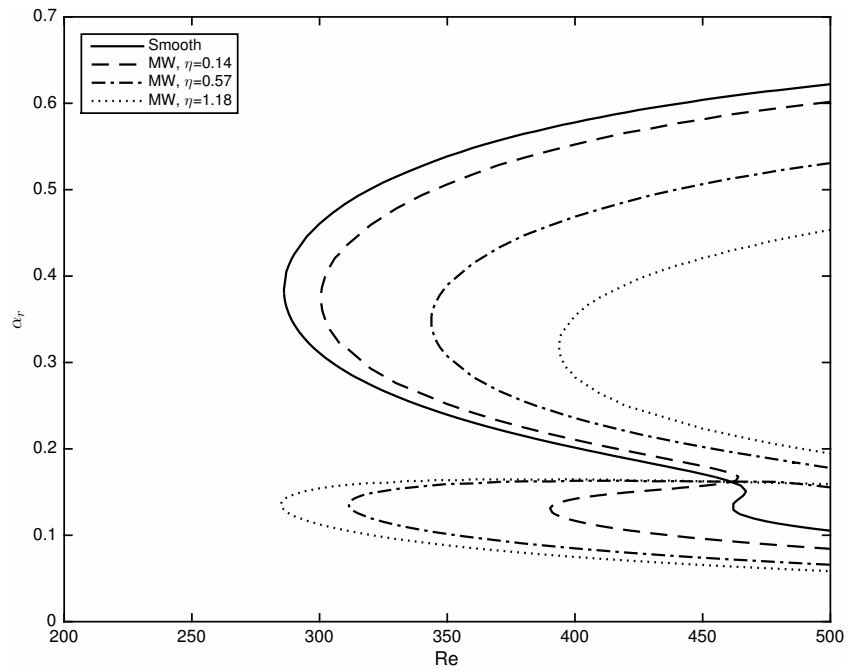

(b) MW model

FIG. 3: The neutral curves in the $R e-\alpha_{r}$ plane resulting from the YHP and MW models with increasing roughness.

As with existing analyses of smooth rotating disks and other related geometries in the literature, two modes are found to determine the convective instability properties of the disturbance modes over rough rotating disks. The Type I mode, appearing as the upper lobe in $R e-\alpha_{r}$ neutral curves, is known to arise from the inflectional nature of the steadyflow profiles, and the Type II mode, appearing as the lower lobe, is known to arise from streamline curvature and Coriolis effects. The results of the spectral code in the smooth case $(a=0=\eta)$ have been compared against those in the literature and the predictions for the critical parameters of the Type I mode are found to be entirely consistent with other published results ${ }^{11,17-22}$. However, as discussed in Appendix A, the literature reports a range of critical values for the Type II mode that appears to suggest sensitivity to the particular calculation method. Our results are at the upper end of those reported in the literature and are very close to those arising from similar codes developed by Appelquist ${ }^{18}$.

Our numerical results have been verified as being independent of the number of Gauss- 


\begin{tabular}{lccc} 
& \multicolumn{3}{c}{ YHP model } \\
& \multicolumn{4}{c}{ Re } & $n$ & $\epsilon$ \\
\hline$a=0$ & $\mathbf{2 8 6 . 1}(461.5)$ & $\mathbf{2 2 . 2}(21.3)$ & $\mathbf{1 1 . 4}(19.2)$ \\
$a=0.1$ & $\mathbf{3 1 1 . 5}(394.4)$ & $\mathbf{2 0 . 7}(16.7)$ & $\mathbf{1 1 . 1}(19.5)$ \\
$a=0.2$ & $426.8(\mathbf{2 8 3 . 3})$ & $20.6(\mathbf{1 1 . 1})$ & $10.8(\mathbf{1 9 . 0})$ \\
$a=0.3$ & $593.9(\mathbf{2 2 0 . 6})$ & $21.8(\mathbf{8 . 5})$ & $11.1(\mathbf{1 8 . 5})$ \\
\multicolumn{4}{c}{ MW model } \\
\multicolumn{5}{c}{$n$} \\
\hline$\eta=0$ & $\mathbf{2 8 6 . 1}(461.5)$ & $\mathbf{2 2 . 2}(21.3)$ & $\mathbf{1 1 . 4}(19.2)$ \\
$\eta=0.14$ & $\mathbf{3 0 0 . 6}(390.3)$ & $\mathbf{1 9 . 6}(17.5)$ & $\mathbf{9 . 8}(16.9)$ \\
$\eta=0.57$ & $343.7(\mathbf{3 1 1 . 5})$ & $15.4(\mathbf{9 . 2})$ & $7.3(\mathbf{1 2 . 3})$ \\
$\eta=1.18$ & $393.8(\mathbf{2 8 4 . 9})$ & $12.4(\mathbf{6 . 3})$ & $5.6(\mathbf{9 . 2})$
\end{tabular}

TABLE II: Critical values of measurable parameters at the onset of instability under both models. Type I and (Type II). Bold text indicates the most dangerous mode in terms of critical Reynolds number.

Lobatto grid points and the upper domain, $z_{\max }$. For example, varying the number of grid points between 50 and 150 leads to a variation in predicted critical Reynolds numbers in the third decimal place only. A similar numerical sensitivity is found when using steady flows obtained with $z_{\max }$ between 15 and 100 .

The neutral curves arising from the analysis of both models are shown in Figure 3. Despite resulting from fundamentally different steady-flow models, both collections of neutral curves display the same qualitative behaviour: the Type I lobe is diminished (both in terms of critical Re and width) with increased roughness, and the Type II mode exaggerated. This is entirely consistent with the results of our previous study of radial isotropic roughness ${ }^{1}$. The results of the YHP model appear much more sensitive to the increased roughness, however this merely reflects the much greater response of the steady flows (as reported in Table I) over the range of $a$ used. Critical parameters at the onset of unstable Type I and Type II modes are given in Table II - we again emphasise that it is inappropriate to make direct numerical comparisons between the two data sets.

The behaviour of the Type II mode under both roughness models is identified as being similar to the effect of wall compliance on this mode, as found by Cooper \& Carpenter ${ }^{17}$. In that study the disk boundary was comprised of a single layer of viscoelastic material free to move under the influence of disturbances in the boundary layer, inducing a disturbance field in the material. For certain levels of wall compliance the Type II lobe of the neutral curve was exaggerated and the critical Re reduced significantly, in exactly the same way as exhibited in this study.

As previously discussed in our detailed analysis of the full $\mathrm{MW}$ model ${ }^{1}$, a consideration of the onset of local absolute instability is important if inferences about delaying the onset of transition with surface roughness are to be made. The effects of surface roughness on the absolute instability are the focus of a separate ongoing study, but preliminary results have shown that roughness acts to delay the onset of absolute instability to significantly higher Re compared to the smooth disk. For example, the onset of absolute instability over a smooth disk ${ }^{12}$ is known to be at around $R e=507$ and initial calculations suggest that the onset of absolute instability is delayed to beyond $R e=700$ for $a=0.3$ in the YHP model, and to beyond $R e=600$ for $\eta=1.18$ in the MW model. We note that in the compliant wall case ${ }^{17}$ it was found that the stabilising effect of wall compliance also suppressed the onset of absolute instability. The similarity between the use of compliant walls to suppress transition and surface roughness is therefore further extended. 


\section{ENERGY ANALYSIS}

Following previous work ${ }^{1,17}$ an integral energy equation for three-dimensional disturbances $(\hat{u}, \hat{v}, \hat{w})$ to the undisturbed three-dimensional boundary-layer flow $(U, V, W)$ is derived in order to extract possible underlying physical mechanisms behind the effects of roughness on the stability of rotating disk boundary-layer flow. Essentially, the energybalance approach enables one to assess the relative influences of the various energy transfer mechanisms affecting the destabilisation of fluid disturbances. The method was used in extensively for the full MW model in our previous publication ${ }^{1}$ and full details are presented there. As demonstrated elsewhere ${ }^{1,17}$, the energy equation that applies to a particular eigenmode is given by

$$
\begin{aligned}
&-2 \alpha_{i}= \underbrace{\left(P_{1}+P_{2}+P_{3}\right)}_{\mathrm{I}}+\underbrace{D}_{\mathrm{II}}+\underbrace{\left(P W_{1}+P W_{2}\right)}_{\mathrm{III}}+ \\
& \underbrace{\left(S_{1}+S_{2}+S_{3}\right)}_{\mathrm{IV}}+\underbrace{\left(G_{1}+G_{2}+G_{3}\right)}_{\mathrm{V}},
\end{aligned}
$$

where the mathematical form of each component, as derived by Cooper \& Carpenter $^{17}$, is given by

$$
\begin{aligned}
& \text { (I) } P_{1}+P_{2}+P_{3}=\int_{0}^{\infty}\left[\left(-\overline{\hat{u} \hat{w}} \frac{\partial U}{\partial z}\right)+\left(-\overline{\hat{v} \hat{w}} \frac{\partial V}{\partial z}\right)+\left(-\overline{\hat{w}^{2}} \frac{\partial W}{\partial z}\right)\right] \mathrm{d} z, \\
& \text { (II) } D=-\int_{0}^{\infty}\left(\overline{\sigma_{i j} \frac{\partial \hat{u}_{j}}{\partial x_{i}}}\right) \mathrm{d} z, \\
& \text { (III) } P W_{1}+P W_{2}=-\int_{0}^{\infty}\left(\frac{\overline{\hat{u} \hat{p}}}{r}\right) \mathrm{d} z+(\overline{\hat{w} \hat{p}})_{\mathrm{w}}, \\
& \text { (IV) } S_{1}+S_{2}+S_{3}=-\left[\overline{\hat{u} \sigma_{31}}+\overline{\hat{v} \sigma_{32}}+\overline{\hat{w} \sigma_{33}}\right]_{\mathrm{w}}, \\
& \text { (V) } G_{1}+G_{2}+G_{3}=-\int_{0}^{\infty} \frac{\partial \bar{K}}{\partial z} W \mathrm{~d} z-\int_{0}^{\infty} \frac{\overline{\hat{u}^{2}}}{\frac{\partial U}{\partial r}} \mathrm{~d} z-\int_{0}^{\infty} \frac{\overline{\hat{v}}^{2} U}{r} \mathrm{~d} z .
\end{aligned}
$$

Here overbars denote a period-averaged quantity, i.e. $\overline{\hat{u}} \hat{v}=\hat{u} \hat{v}^{*}+\hat{u}^{*} \hat{v}\left({ }^{*}\right.$ indicates the complex conjugate) and w subscripts denote quantities evaluated at the wall. Furthermore, $K=\frac{1}{2}\left(\hat{u}^{2}+\hat{v}^{2}+\hat{w}^{2}\right)$, and $\sigma_{i j}$ are the viscous stress terms

$$
\sigma_{i j}=\frac{1}{R e}\left(\frac{\partial \hat{u}_{i}}{\partial x_{j}}-\frac{\partial \hat{u}_{j}}{\partial x_{i}}\right) .
$$

Physically, the terms in equation (7) as identified as follows

(I) the Reynolds stress energy production terms, $\left\{P_{i}\right\}$

(II) the viscous dissipation energy removal term, $D$

(III) pressure work terms, $\left\{P W_{i}\right\}$

(IV) contributions from work done on the wall by viscous stresses, $\left\{S_{i}\right\}$

(V) terms arising from streamline curvature effects and the three-dimensionality of the mean flow, $\left\{G_{i}\right\}$.

Terms that are positive contribute to the energy production and those which are negative remove energy from the system. A particular eigenmode is amplified when energy production outweighs the energy dissipation in the system, which is consistent with the instability criteria $\left(\alpha_{i}<0\right)$ used to obtain the neutral curves in $\S I I I$.

Calculations have been carried out for both roughness models, and for both the Type I and II modes at $R e=400$. The corresponding growth rates are shown in Figure 4. This emphasises the stabilising effect of roughness on the Type I mode, the destabilising effect 


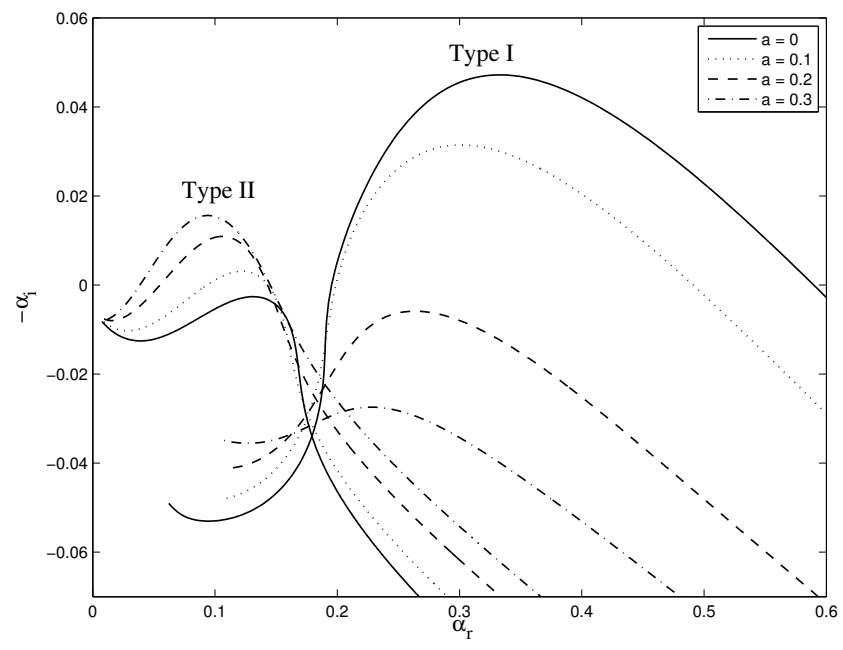

(a) YHP model

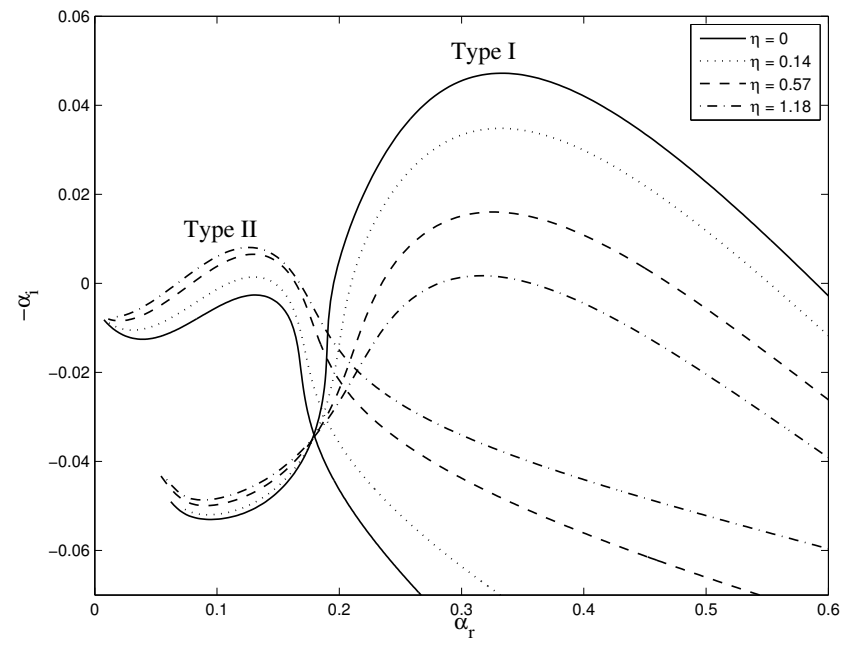

(b) MW model

FIG. 4: Type I and II growth rate curves at $R e=400$.

on the Type II mode and the stronger effect on both of these modes for the YHP model. For the MW model the amplification of the Type II mode is more modest, even though the Type II lobe of the neutral curve shows similar augmentation to the YHP case, and the stabilising effect on the Type I mode is not quite so strong.

By calculating all terms in the energy equation (7), it is possible to identify where the effects of roughness are the greatest. Given the boundary conditions for the YHP model some terms in the energy equation are identically zero $\left(P W_{2}, S_{1}, S_{2}, S_{3}\right)$. The results of the energy balance for the three roughness values $a=0.1,0.2$ and 0.3 are compared to those for a smooth disk $(a=0)$ in Figure 5 . For both modes the main contributors are energy production by the Reynolds stress $\left(P_{2}\right)$ and conventional viscous dissipation $(D)$. Terms $P_{1}, P_{3}, P W_{1}$ and $G_{2}$ are found to be negligible and the geometric terms $G_{1}$ and $G_{3}$ remove energy from the system. The strongly stabilising effect of roughness on the Type I mode is manifested in a striking reduction in $P_{2}$ and a slight increase in viscous dissipation. Conversely, the growth with roughness of the Type II mode arises from a net increase in energy production through increased Reynolds stress alongside with a reduction in viscous 


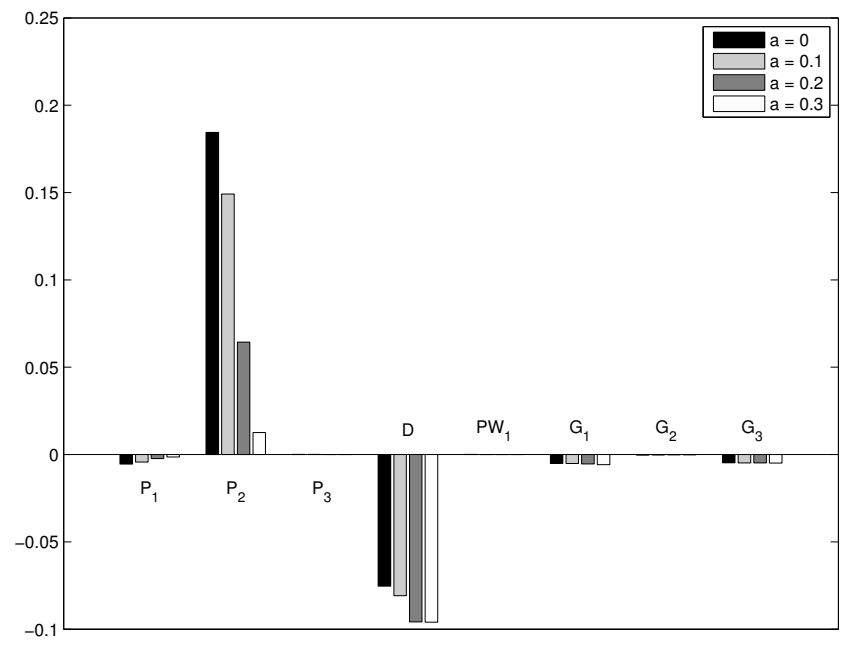

(a) Type I mode with $n=28$.

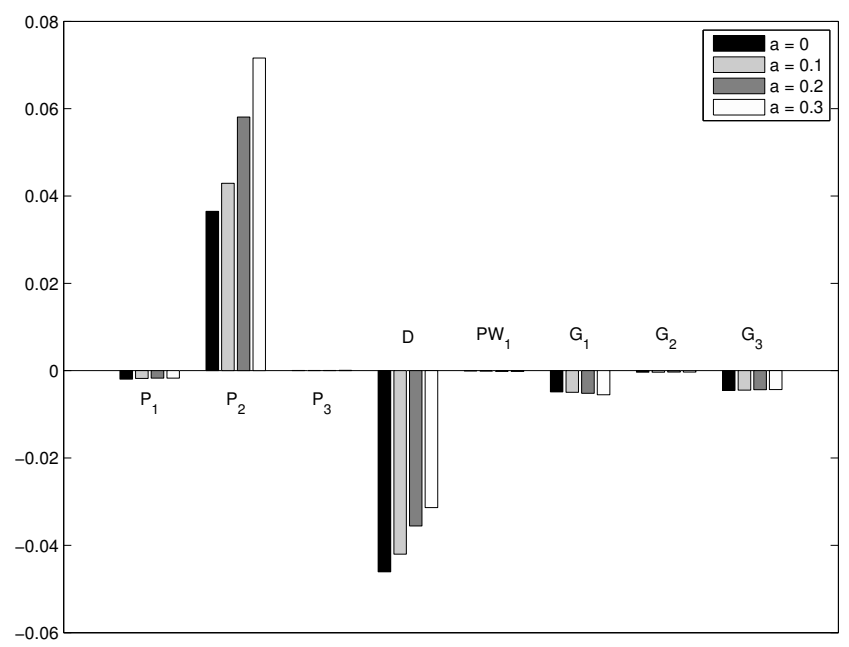

(b) Type II mode with $n=12$.

FIG. 5: Results of energy analysis for YHP model at $R e=400$.

dissipation.

The form of the eigenfunctions (or disturbance velocity profiles) provides some explanation for the above trends. The dominant eigenfunction is the azimuthal perturbation velocity, $v$, which contributes to the dominant energy production term $P_{2}$. Figure 6 shows the magnitude of the $v$-profile for the roughness cases considered. In the case of the Type II mode the effect of roughness is seen through the eigenfunctions extending further in to the boundary layer and the profiles becoming more stretched out as roughness is increased. Corresponding results for the Type I mode show that the general form of the disturbance profile is preserved in this case, with the profile being merely translated slightly further into the boundary layer as roughness is increased. The dramatic reduction in $P_{2}$ in this case results from a strong reduction in the amplitude of the normal velocity, $w$, as roughness increases. As explained elsewhere ${ }^{1,17}$, the viscous dissipation term $D$ is dominated by the term $\overline{\sigma_{32} \partial \hat{v} / \partial z}$ so that $D \approx-(2 / R e) \int|\overline{\partial \hat{v} / \partial z}|^{2} \mathrm{~d} z$. The broadening of the $v$ velocity profile for the Type II mode has the effect of decreasing $D$. The effect of roughness on the distribution for $D$ is less significant for the Type I mode. 


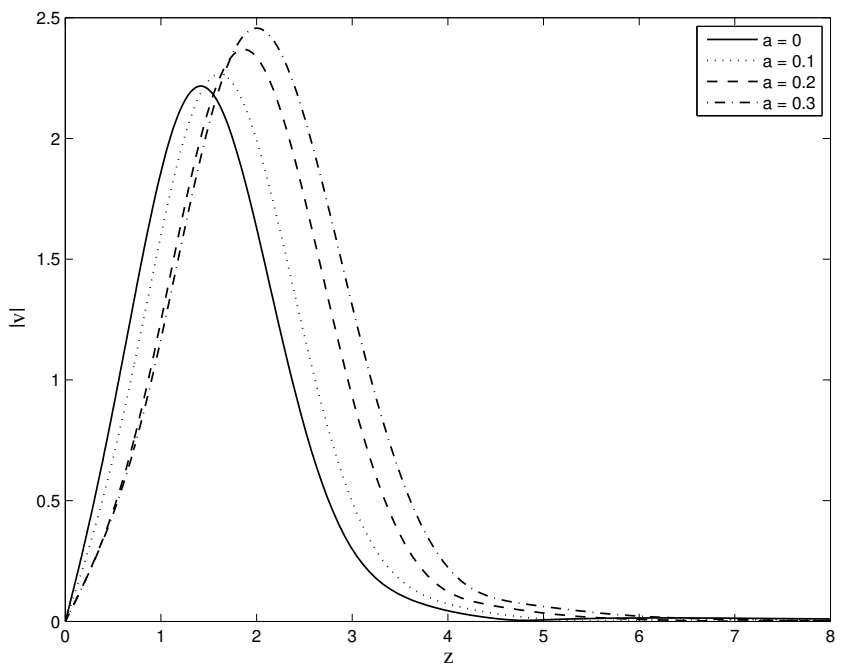

(a) Type I mode with $n=28$.

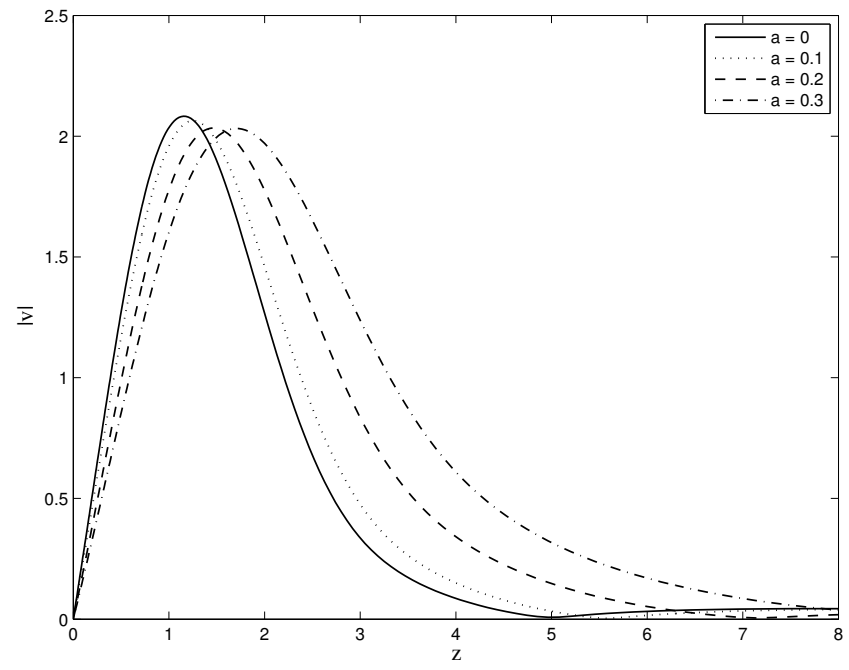

(b) Type II mode with $n=12$.

FIG. 6: Profiles for azimuthal perturbation velocity in YHP model.

In summary, the Type II disturbances generally extend further into the boundary layer than the Type I disturbances. The further stretching of the disturbance profile as roughness is increased, together with the thickening of the boundary layer with roughness, would appear to contribute to the augmentation of the Type II mode.

Results of the energy balance calculation for the MW model are shown in Figure 7 . Again both modes are dominated by contributions from $P_{2}$ and $D$. The main difference from the YHP model in the case of the Type II mode is that, although the Reynolds stress energy production term increases as before, the MW model also shows an increase in viscous dissipation (opposite to YHP) which would account for the more modest growth observed in Figure 4. The Type I mode shows a less pronounced decrease in $P_{2}$, but more viscous dissipation compared to the YHP model.

Figure 8(b) shows a similar stretching of the Type II eigenmodes to the YHP case. Since there is no increase in the boundary-layer thickness for the MW steady flow, the fluid disturbances do not extend quite as far into boundary layer as for the YHP case. The Type 


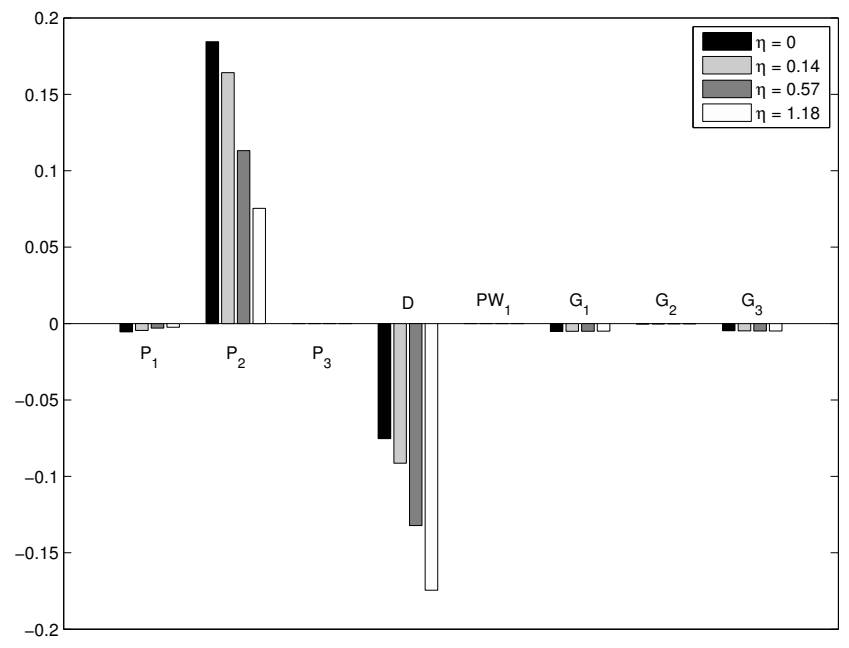

(a) Type I mode with $n=28$.

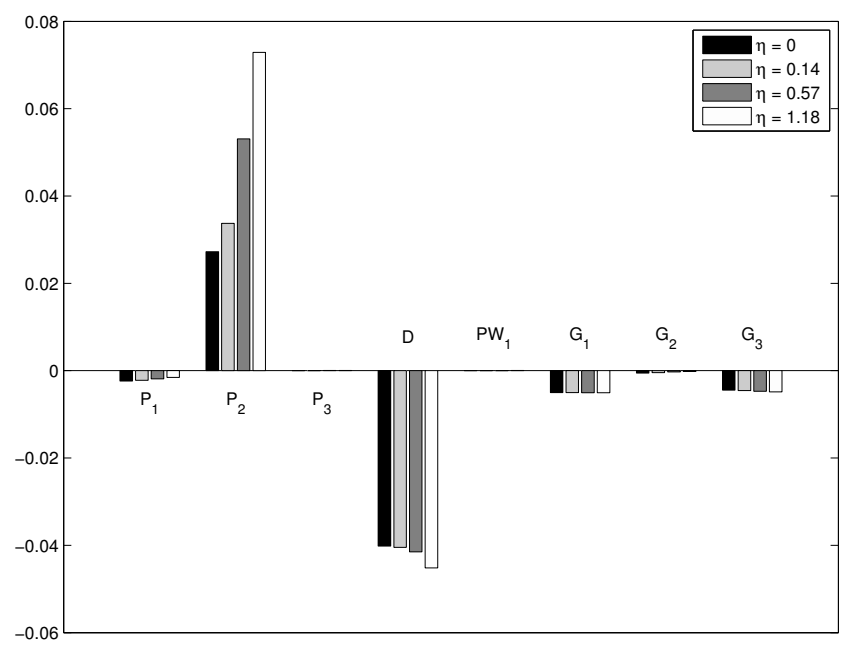

(b) Type II mode with $n=8$.

FIG. 7: Results of energy analysis for MW model at $R e=400$.

I disturbance profiles are very similar to those for the YHP model.

\section{CONCLUSION}

We have summarised and discussed the results of a theoretical study investigating the effects of radial anisotropic surface roughness resulting from concentric grooves on the stability of the von Kármán boundary-layer flow over a rotating disk. Our theoretical analysis was based on the two alternative modelling approaches suggested by Yoon et al. ${ }^{10}$ and by Miklavčič \& Wang ${ }^{2}$ for modelling the steady boundary-layer flow over a rough rotating disk. Whereas the former approach describes roughness by explicitly prescribing a particular geometry for the surface roughness, the latter models roughness by a modification of the no-slip boundary conditions at the disk surface.

As regards practical applications, the most significant overall result of our study is that we have been able to confirm our conclusion from Ref. [1], by means of a different modelling 


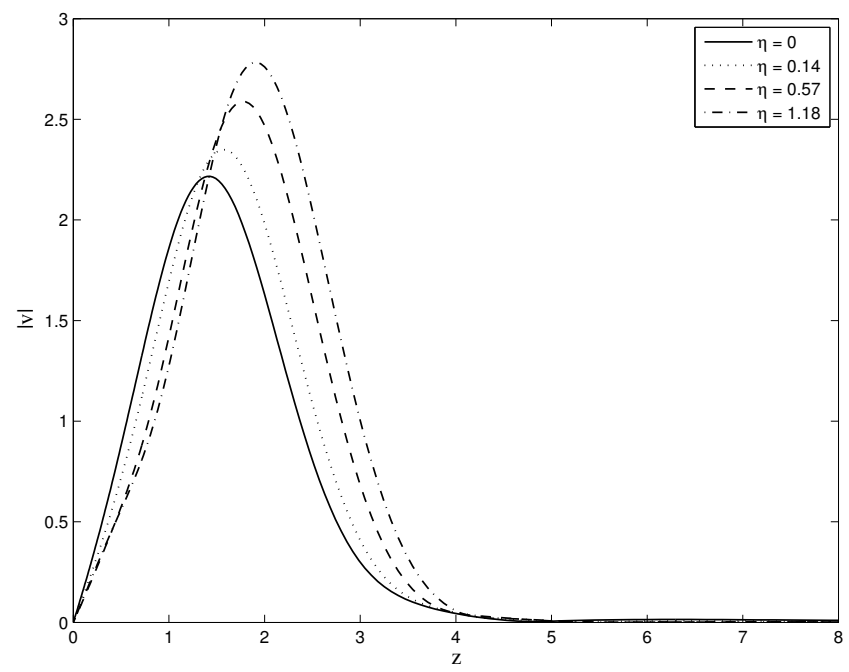

(a) Type I mode with $n=28$.

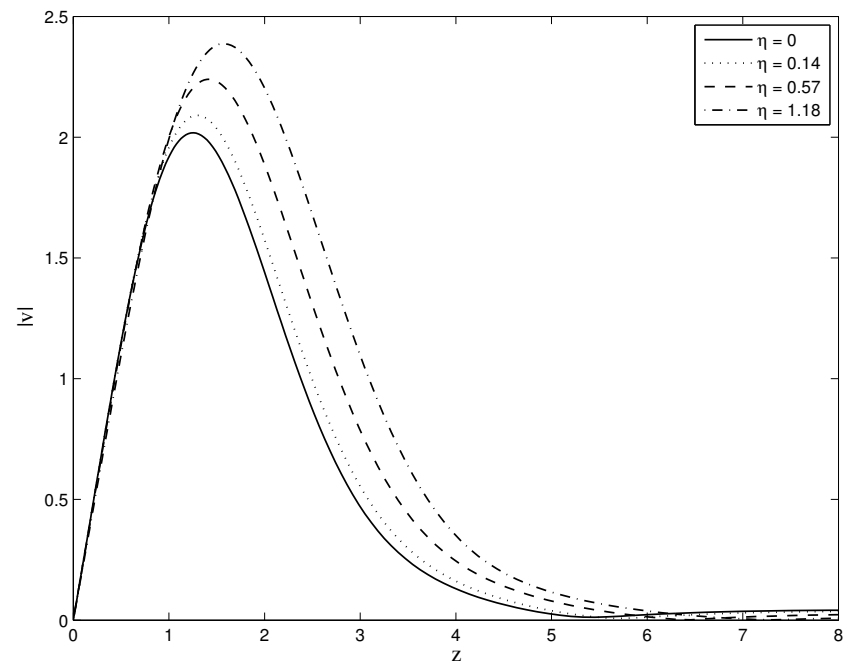

(b) Type II mode with $n=8$.

FIG. 8: Profiles for azimuthal perturbation velocity in MW model.

approach, that surface roughness is expected to stabilise the Type 1 instability mode of the rotating-disk boundary layer. This means that the stabilisation is not just an artefact arising from the particular methodologies associated with the MW model. Therewith it now appears substantially more likely that it may indeed be possible to exploit surface roughness in practice for focussed, theory-led approaches to designing the right sort of roughness ${ }^{8}$ for the purpose of laminar-flow control by devising optimisation strategies employing our suggested energy analysis.

Nevertheless, it is evidently still far from clear which of the two approaches adopted to model surface roughness will ultimately reveal itself as better suited to correctly describe experimental data when these become available. Only detailed experimental investigations will eventually enable establishing if one of the two models is indeed representative of distributed roughness or whether modifications, or even entirely different alternative approaches, have to be sought. If future experiments reveal that neither one of the two models is entirely satisfactory then the inter-comparison of these first results described here 
will assist in pointing a way forward to systematic strategies for adapting the modelling methodologies taking into account the different qualitative behaviours found in our current investigation.

In relation to the von Kármán similarity solution for flow over smooth disks, the two methodologies for modelling roughness have resulted in qualitatively different modifications of the steady-flow base profiles. However, these profiles are underlying the subsequent stability analysis for the boundary-layer flow. In particular, it was found, for instance, that predictions of the azimuthal velocity profile based on the YHP methodology approach the von Kármán solution near the disk surface whereas the predictions of the MW model are more similar to the von Kármán profiles at intermediate heights above the disk. The situation was somewhat more complex for the radial and the wall-normal component. While we have here not studied how such differences lead to specific variations in the predicted neutral stability, it is clear that it must be certain aspects of these small-scale differences that govern the global stability behaviour. Nevertheless, it was found that, overall, the effects arising when following the YHP model are somewhat more pronounced than when using the MW approach. This apparent sensitivity may, however, be a consequence of the difficulty associated with defining 'equivalent' levels of roughness between the two models which do not allow direct quantitative comparisons due to the different nature of the two approaches.

The results obtained from our linear stability analysis were thereafter reconfirmed by an energy analysis consistent with that described in other published studies ${ }^{1,17}$. The energy analysis has revealed that for both the YHP and the MW approach, and for both the Type I and Type II instability modes, the main contributors to the energy balance are the energy production by the Reynolds stresses and conventional viscous dissipation. For the Type I mode dissipation increases with the roughness level and the increase is more pronounced for the MW model than for the YHP model. For the Type I mode Reynolds-stress energy production decreases with the roughness level and the decrease is more pronounced for the YHP model than for the MW model. Thus, in summary, increased energy dissipation and decreased energy production by Reynolds stresses implies a stabilisation of the Type I mode by increasing roughness levels.

For the Type II mode Reynolds-stress energy production increases with the roughness level and the increase is slightly less pronounced for the YHP model than for the MW model. The main qualitative difference is observed for the energy dissipation of the Type II mode. For the Type II mode energy dissipation decreases with the roughness level for the YHP model whereas there is a slight increase under the MW model. Yet, the overall increased Reynolds-stress energy production and decreased energy dissipation result in a destabilisation of the Type II mode for both the YHP and the MW model.

Our study suggests that the beneficial stabilisation of the Type I mode by concentric roughnesses becomes suppressed when the Type II mode is destabilised as it moves upstream and eventually becomes the critical mode at the lowest Reynolds number. This is consistent with our previous results ${ }^{1}$. The results of the energy analysis imply that the dissipation of the Type II mode is sensitive to the precise form of the steady-flow base profile. Consequently, maximising dissipation by an appropriately designed surface-roughness pattern, that leads to the energetically optimal base profile, can theoretically lead to an overall beneficial stabilisation of the Type II mode. Provided that the Type I mode is not adversely affected, this could result in a boundary-layer-transition delay and drag reduction. This points to a possible way forward for exploiting the beneficial stabilising effects of radial anisotropic roughness on the Type I mode in future drag-reduction techniques relying on transition delay for boundary layers with a cross-flow component.

SJG is supported by a Senior Research Fellowship of the Royal Academy of Engineering, funded by the Leverhulme Trust. MÖ wishes to acknowledge financial support from Republic of Turkey Ministry of National Education. Useful conversations with Ellinor Appelquist of KTH are gratefully acknowledged. 
Type I mode

\begin{tabular}{|c|c|c|c|c|}
\hline & $R e$ & $n$ & $\epsilon$ & $\alpha_{r}$ \\
\hline Malik $^{11}$ & 285.36 & 22.14 & 11.4 & 0.38482 \\
\hline Balakumar \& Malik $^{19}$ & 286.05 & 22.26 & 11.4 & 0.38643 \\
\hline Cooper \& Carpenter ${ }^{17}$ & 285.36 & 22.13 & 11.4 & 0.38451 \\
\hline Turkilmazoglu \& Gajjar ${ }^{20}$ & 286.05 & 22.15 & 11.4 & 0.38407 \\
\hline Thomas $^{22}$ & 290.00 & 22.33 & 11.5 & 0.37790 \\
\hline Garrett et $a l .{ }^{21}$ & 285.36 & 22.20 & 11.4 & 0.38537 \\
\hline Appelquist $^{18}$ (shooting) & 286.05 & 22.20 & 11.4 & 0.38338 \\
\hline Appelquist $^{18}$ (spectral) & 286.05 & 22.21 & 11.4 & 0.38527 \\
\hline This paper & 286.05 & 22.16 & 11.4 & 0.38419 \\
\hline
\end{tabular}

\begin{tabular}{lcccc} 
& \multicolumn{4}{c}{ Type II mode } \\
& $R e$ & $n$ & $\epsilon$ & $\alpha_{r}$ \\
\hline Malik $^{11}$ & 440.88 & 20.60 & 19.5 & 0.13228 \\
Balakumar \& Malik $^{19}$ & 451.40 & 20.95 & 19.5 & 0.13109 \\
Cooper \& Carpenter $^{17}$ & 440.87 & 20.55 & 19.5 & 0.13159 \\
Turkilmazoglu \& Gajjar $^{20}$ & 453.76 & 21.16 & 19.4 & 0.13198 \\
Thomas $^{22}$ & 451.00 & 20.93 & 19.2 & 0.13360 \\
Garrett et al. $^{21}$ & 450.95 & 20.90 & 19.5 & 0.13067 \\
Appelquist $^{18}$ (shooting) & 452.97 & 21.20 & 19.4 & 0.13227 \\
Appelquist & & & & \\
Appectral) & 460.90 & 21.23 & 19.2 & 0.13158 \\
This paper & 461.51 & 21.33 & 19.2 & 0.13260
\end{tabular}

TABLE III: Critical values of measurable parameters at the onset of Type I and Type II instabilities as reported in the literature.

\section{Appendix A: Literature survey of critical instability parameters for smooth disks}

Table III summarises published critical values for the onset of the Type I and Type II convective modes of instability in the von Kármán boundary layer over a smooth disk. We note a relatively close spread of values for the onset of the Type I mode but a larger spread for the onset of the Type II mode. In particular, the results appear to cluster around either $R e \approx 440,450$ or 460 . The results of this current study are at the upper end of the range reported in the literature.

We suggest that the spread of values in the Type II results can be attributed to an apparent sensitivity to the formulation of the solution method. This is consistent with the comment of Balakumar \& Malik ${ }^{19}$ who attribute their discrepancy with Malik's ${ }^{11}$ earlier result to the use of a perturbation system expressed in terms of primitive variations (as we do here) rather then a transformed vorticity formation. No such sensitivity appears to exist for the Type I results.

${ }^{1}$ Cooper, A.J., Harris, J.H., Garrett, S.J., Thomas, P.J. \& Özkan, M. 2015 The effect of anisotropic and isotropic roughness on the convective stability of the rotating disk boundary layer, Phys. Fluids, 27, 014107.

${ }^{2}$ Miklavčič, M. \& Wang, C.Y. 2004. The flow due to a rough rotating disk. Z. angew. Math. Phys., 54, 235-246.

${ }^{3}$ Kármán, von Th. 1921 Ueber laminare und turbulente Reibung, Z. Angew. Math. Mech. 1, 233-252.

${ }^{4}$ Lingwood, R.J. \& Alfredsson, P.H. 2015 Instabilities of the von Kármán boundary layer, Appl. Mech. Rev. 67, 030803.

${ }^{5}$ Reed, H.L. \& Saric, W.S. 1989 Stability of three-dimensional boundary layers, Annu. Rev. Fluid Mech. 21, 235-284.

${ }^{6}$ Saric, W.S., Reed, H.L. \& White, E.W 2003 Stability and transition of three-dimensional boundary layers, Annu. Rev. Fluid Mech., 35, 413-440. 
${ }^{7}$ Sirovich, L. \& Karlsson, S. 1997 Turbulent drag reduction by passive mechanisms, Nature 388, 753-755. ${ }^{8}$ Carpenter, P. W. 1997 The right sort of roughness, Nature 388, 713-714.

${ }^{9}$ Choi, K. S. 2006 The rough with the smooth, Nature 440, 754.

${ }^{10}$ Yoon, M.S., Hyun, J.M. \& Park, J.S 2007 Flow and heat transfer over a rotating disk with surface roughness.International Journal of Heat and Fluid Flow, 28(2), 262-267.

${ }^{11}$ Malik, M. R. 1986 The neutral curve for stationary disturbances in rotating-disk flow. J. Fluid Mech. 164, 275-287.

${ }^{12}$ Lingwood, R.J. 1995 Absolute instability of the boundary layer on a rotating disk, J. Fluid Mech. 299, 17.

${ }^{13}$ Banks, W. H. H. 1965 The boundary layer on a rotating sphere. Q. J. Mech. Appl. Math 18, 443-454

${ }^{14}$ Garrett, S.J. \& Peake, N. 2002 The stability and transition of the boundary layer on a rotating sphere, J. Fluid Mech. 456, 199-218.

${ }^{15}$ Harris, J.H. 2013 Stability of the flow over a rough rotating disk (Doctoral dissertation). University of Warwick.

${ }^{16}$ Lingwood, R.J. \& Garrett, S.J. 2011 The effects of surface mass flux on the instability of the BEK system of rotating boundary layer flows, European J. Mech. B 30, 299-310.

${ }^{17}$ Cooper, A.J. \& Carpenter, P.W. 1997 The stability of rotating-disc boundary-layer flow over a compliant wall. Part 1. Type I and II instabilities, J. Fluid Mech. 350, 231-259.

${ }^{18}$ Appelquist, E. 2014 Direct numerical simulations of the rotating-disk boundary-layer flow. Licentiate thesis, Royal Institute of Technology, KTH Mechanics, ISBN: 978-91-7595-202-4

${ }^{19}$ Balakumar, P. \& Malik, M. 1990 Travelling disturbances in rotating-disk flow, Theoret. Comput. Fluid Dyn. 2, 125, 137.

${ }^{20}$ Turkilmazoglu, M. \& Gajjar, J.S.B. 1998 Convective and absolute instability in the incompressible boundary layer on a rotating-disk. Technical report, Department of Mathematics, University of Manchester.

${ }^{21}$ Garrett, S.J., Hussain, Z. \& Stephen, S.O. 2009 The cross-flow instability of the boundary layer on a rotating cone, J. Fluid Mech. 622, 209-232.

${ }^{22}$ Thomas, C. 2007 Numerical simulations of disturbance development in rotating boundary-layers, PhD thesis, Cardiff University. 